Paediatr. Paedolog. 2020 · 55:160-161 https://doi.org/10.1007/s00608-020-00826-w

(c) Springer-Verlag GmbH Austria, ein Teil von Springer Nature 2020

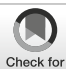

\section{Daniela Karall · Sabine Scholl-Bürgi}

Department für Kinder- und Jugendheilkunde, Universitätsklinik für Pädiatrie I, Bereich Angeborene Stoffwechselstörungen, Medizinische Universität Innsbruck, Innsbruck, Österreich

\title{
Bilanz ziehen Zahlen, Daten, Fakten
}

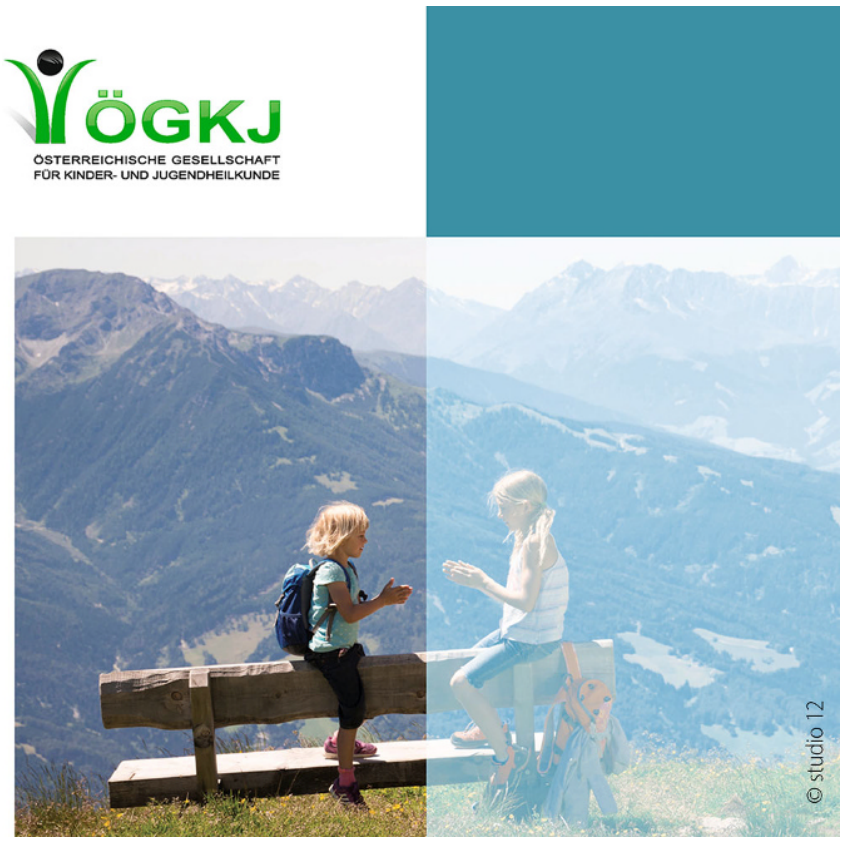

Das Thema der heurigen Jahrestagung „Bilanz ziehen - Zahlen, Daten, Fakten“ zielt darauf ab, eine Standortbestimmung der Pädiatrie zu ermöglichen - anhand quantifizierbarer und bewertbarer Parameter. Als Pädiaterinnen und Pädiater sehen wir uns gelegentlich damit konfrontiert, dass wir als „Ärzte für die Kleinen“ betrachtet werden. Dabei ist die Pädiatrie wohl das letzte Fach, das tatsächlich sehr breit aufgestellt ist, da es sowohl häufige, wie auch seltene Krankheitsbilder betreut und sich nicht durch ein Organ, sondern durch den Alterszeitraum von 0 bis 18 Jahren definiert.

Das Motto der diesjährigen 58. Jahrestagung der Österreichischen Gesellschaft für Kinder- und Jugendheilkunde (ÖGKJ), die vom 24. bis 26. September 2020 in Innsbruck stattfinden wird, soll die Weitergabe von Fakten verdeutlichen, aber auch zum Nachdenken anregen.
Als der Titel der Jahrestagung festgelegt wurde, war die Welt um uns herum noch vollkommen anders - keiner wusste damals, dass COVID-19/SARS CoV2 so bestimmend werden würde. In Anbetracht der Corona-Pandemiemaßnahmen waren wir gefordert, uns ganz neu mit der Struktur der heurigen Jahrestagung auseinanderzusetzen. Längere Zeit war es unsicher, ob die Tagung überhaupt als Präsenztagung würde stattfinden können. Wir haben in der Zeit von Corona vieles über die Möglichkeiten der Technik gelernt. Allerdings ersetzen virtuelle Tagungen nicht vollständig den persönlichen Kontakt und Austausch untereinander. Deswegen sind wir froh, dass es - unter Beachtung der gegebenen behördlichen Bestimmungen - nach heutigem Stand (15.08.2020) möglich sein wird, die Jahrestagung fast „wie immer“ abzuhalten.
Das Hauptthema 2020 ist „Bilanz ziehen" - Zahlen, Daten, Fakten in der Pädiatrie. Wir sind in der glücklichen Lage, dass die Jahrestagung ein nationales Ereignis ist und daher Einreisebestimmungen oder -beschränkungen für Redner sich nicht negativ auswirken. Aus dem reichhaltigen Fundus an Kolleginnen und Kollegen, die Expertise in den unterschiedlichsten Bereichen haben, werden insgesamt in vier Plenarsitzungen Vorträge zu den Themen Fazit aus epidemiologischen Erhebungen, Pädiatrie in einer geänderten Zeit (Teil 1 und 2) und Update zu neuen Therapien halten. Daneben bestreiten sie weitere sechs Parallelsitzungen. Aus den eingereichten wissenschaftlichen Beiträgen (Abstracts) konnten neben einer 94 Poster umfassenden Ausstellung weitere vier Parallelsitzungen mit insgesamt 30 Kurzvorträgen gestaltet werden, drei davon als Late Breaking

\begin{tabular}{|l}
\hline Bitte beachten \\
\hline Dem Thema Nachhaltigkeit/Umweltschutz \\
wird bei der ÖGKJ-Jahrestagung mehr \\
Gewicht gegeben werden. Die Tagung ist als \\
Green Event zertifiziert. \\
Das heißt, dass einige Punkte konkret gelten: \\
1. Auf den Druck des Jahrestagungspro- \\
gramms und des Abstract-Bands wird \\
verzichtet. Beide werden als PDF-File \\
vor der Jahrestagung auf der Homepage \\
(https://www.oegkj-jahrestagung.at/) und \\
auch in der Jahrestagungs-APP verfügbar \\
sein. Wer ein papierenes ausgedrucktes Pro- \\
gramm oder einen Abstract-Band wünscht, \\
soll sie bitte selbst zur Tagung mitbringen! \\
2. Auf eine Kongresstasche mit Werbeeinla- \\
gen wird verzichtet. \\
3. Um Anreise mit öffentlichen Verkehrsmit- \\
teln wird gebeten. \\
4. Regionales und saisonales Catering ist \\
vorgesehen.
\end{tabular}


News (mit Einreichfrist der Abstracts bis 10.09.2020).

Den Abschluss der Jahrestagung bilden am Samstag, den 26. September, halb- und ganztägige Workshops zu unterschiedlichsten Themenbereichen.

Der Facharztprüfungs-Vorbereitungskurs wird am 23.09., 12:00-18:30, und 24.09, 09:00-12:00, an der Klinik Innsbruck, Anichstraße 35, stattfinden - mit den Themenschwerpunkten Nephrologie, Pulmonologie und Infektiologie. Die Anmeldung ist bis 20.09.2020 möglich.

Am Freitag, 25.09., tagen parallel zur ÖGKJ auch:

1. der Berufsverband Kinderkrankenpflege Österreich (BKKÖ) und

2. die Forschungszentren Medical Humanities, Medizin- und Gesundheitsrecht zum Thema KIND.RECHT.MEDIZIN

Die jeweils aktualisierten Informationen zum Programm sind auf der Homepage www.oegkj-jahrestagung.at oder auch www.paediatrie.at $z u$ finden.

Wir freuen uns, Sie hoffentlich bald in Innsbruck begrüßen zu können!

Ihre

Daniela Karall

ÖGKJ Präsidentin

Sabine Scholl-Bürgi

ÖGKJ erste Sekretärin

und Tagungsorganisator*innen

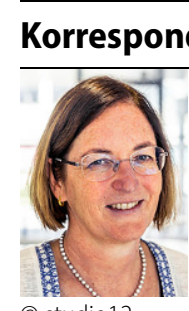

(c) studio12

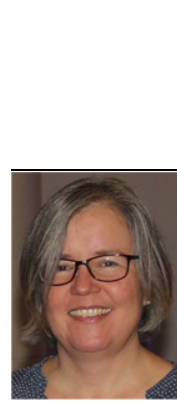

(c) S. Scholl-Bürg

S. Scholl-Büri

Ao. Univ.-Prof. Dr.

Daniela Karall, IBCLC

Department für Kinderund Jugendheilkunde,

Universitätsklinik für Pädiatrie

I, Bereich Angeborene

Stoffwechselstörungen,

Medizinische Universität

Innsbruck

Anichstraße 35, 6020 Inns-

bruck, Österreich

daniela.karall@i-med.ac.at

Priv.-Doz. Dr. Sabine SchollBürgi

Department für Kinderund Jugendheilkunde, Universitätsklinik für Pädiatrie

I, Bereich Angeborene

Stoffwechselstörungen,

Medizinische Universität Innsbruck

Anichstraße 35, 6020 Inns-

bruck, Österreich

Interessenkonflikt. D. Karall gibt an, dass kein Interessenkonflikt besteht.

Hinweis des Verlags. Der Verlag bleibt in Hinblick auf geografische Zuordnungen und Gebietsbezeichnungen in veröffentlichten Karten und Institutsadressen neutral.
Hier steht eine Anzeige. Springer 